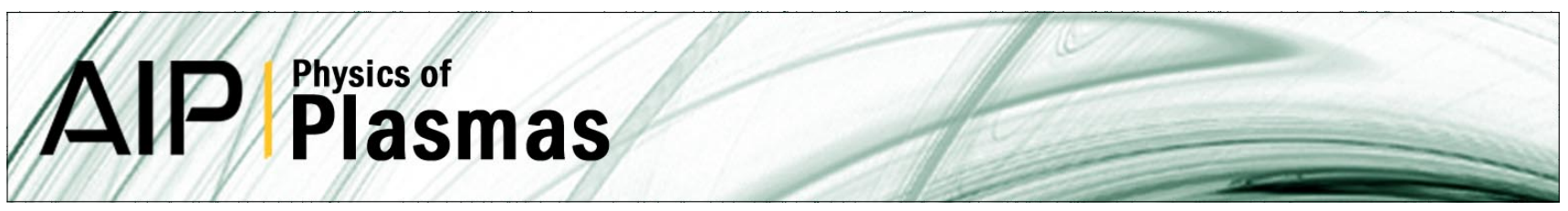

\title{
Influence of the lower hybrid drift instability on the onset of magnetic reconnection
}

Paolo Ricci, J. U. Brackbill, W. Daughton, and Giovanni Lapenta

Citation: Phys. Plasmas 11, 4489 (2004); doi: 10.1063/1.1778744

View online: http://dx.doi.org/10.1063/1.1778744

View Table of Contents: http://pop.aip.org/resource/1/PHPAEN/v11/i9

Published by the American Institute of Physics.

\section{Related Articles}

Inclusion of diamagnetic drift effect in the matching method using finite-width inner region for stability analysis of magnetohydrodynamic modes

Phys. Plasmas 19, 102511 (2012)

On the heterogeneous character of the heartbeat instability in complex (dusty) plasmas

Phys. Plasmas 19, 103701 (2012)

Three-dimensional modeling and analysis of a high energy density Kelvin-Helmholtz experiment

Phys. Plasmas 19, 092112 (2012)

Effect of ion-to-electron mass ratio on the evolution of ion beam driven instability in particle-in-cell simulations Phys. Plasmas 19, 092111 (2012)

Experimental observations of turbulent mixing due to Kelvin-Helmholtz instability on the OMEGA Laser Facility Phys. Plasmas 19, 092702 (2012)

\section{Additional information on Phys. Plasmas}

Journal Homepage: http://pop.aip.org/

Journal Information: http://pop.aip.org/about/about_the_journal

Top downloads: http://pop.aip.org/features/most_downloaded

Information for Authors: http://pop.aip.org/authors

\section{ADVERTISEMENT}

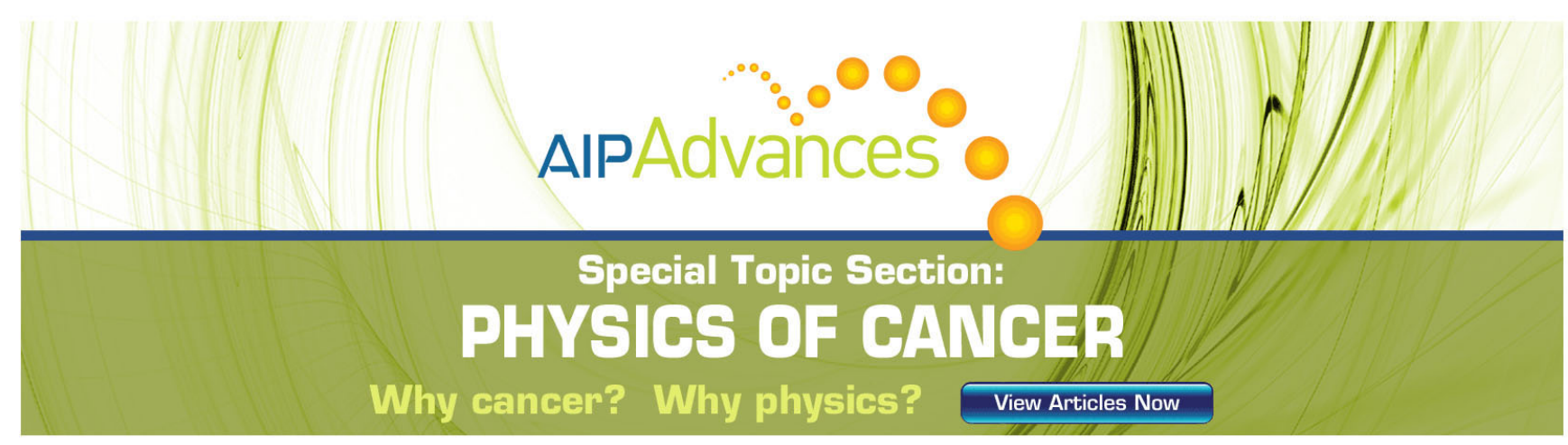




\title{
Influence of the lower hybrid drift instability on the onset of magnetic reconnection
}

\author{
Paolo Riccia \\ Istituto Nazionale per la Fisica della Materia (INFM), Unità del Politecnico di Torino, \\ Corso Duca degli Abruzzi 24-10129 Torino, Italy \\ and Dipartimento di Energetica, Politecnico di Torino, Torino, Italy \\ J. U. Brackbill ${ }^{\text {b) }}$ and W. Daughton ${ }^{\text {c) }}$ \\ Los Alamos National Laboratory, Los Alamos, New Mexico 87545 \\ Giovanni Lapenta ${ }^{\text {d) }}$ \\ Istituto Nazionale per la Fisica della Materia (INFM), Unità del Politecnico di Torino, \\ Corso Duca degli Abruzzi 24-10129 Torino, Italy \\ and Los Alamos National Laboratory, Los Alamos, New Mexico 87545
}

(Received 9 March 2004; accepted 9 June 2004; published online 26 August 2004)

\begin{abstract}
Two-dimensional and three-dimensional kinetic simulation results reveal the importance of the lower-hybrid drift instability (LHDI) to the onset of magnetic reconnection. Both explicit and implicit kinetic simulations show that the LHDI heats electrons anisotropically and increases the peak current density. Linear theory predicts these modifications can increase the growth rate of the tearing instability by almost two orders of magnitude and shift the fastest growing modes to significantly shorter wavelengths. These predictions are confirmed by nonlinear kinetic simulations in which the growth and coalescence of small scale magnetic islands leads to a rapid onset of large scale reconnection. (C) 2004 American Institute of Physics. [DOI: 10.1063/1.1778744]
\end{abstract}

\section{INTRODUCTION}

Understanding the onset of magnetic reconnection remains an open question. Reconnection is observed in a great variety of systems, from special purpose laboratory experiments, ${ }^{1-4}$ to fusion devices ${ }^{5}$ and space $^{6-8}$ and astrophysical plasmas. ${ }^{9-11}$ Our focus is on reconnection in the magnetosphere due to interactions with the solar wind. Reconnection develops mainly in current sheets at the magnetopause ${ }^{7}$ and in the tail. ${ }^{6}$

In both situations, the field can be approximated by a Harris current sheet. ${ }^{12}$ In fact, a reference configuration for such plasmas is proposed, the so-called geophysical environment magnetic (GEM) reconnection challenge, to bring uniformity, repeatability, and ease of intercomparison among various models (see Ref. 13 and references therein).

The original GEM challenge is initialized with a large magnetic island (perturbation of $10 \%$ amplitude) so that both magnetohydrodynamic (MHD) modeling and kinetic simulations can study reconnection, after reconnection starts. Thus, the GEM challenge bypasses reconnection onset and allows researchers to study the physics of reconnection in its nonlinear phase. In our simulations, we consider a GEM equilibrium in which an initial perturbation is absent so that we can study reconnection onset.

There have been many previous studies of reconnection onset. Among kinetic studies, which are most relevant to collisionless magnetospheric plasmas where the current sheet

\footnotetext{
${ }^{a)}$ Electronic mail: paolo.ricci@polito.it

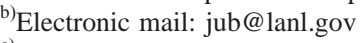

${ }^{c}$ Electronic mail: daughton@lanl.gov

${ }^{\mathrm{d})}$ Electronic mail: lapenta@lanl.gov
}

thicknesses are of the order of the ion gyroradius or ion skin depth, the onset of reconnection has been long attributed to the tearing instability. ${ }^{14-16}$

However, the tearing instability saturates at low levels: For typical magnetospheric configurations, tearing islands are predicted to saturate when the width approaches the electron skin depth. ${ }^{17}$ These are far too small to trigger reconnection by any of the recently investigated, nonlinear mechanisms for fast reconnection. The GEM challenge, with its large initial perturbation, produces a large enough starting island size to decouple electron and ion motion. This allows fast reconnection through Hall or whistler physics reconnection. ${ }^{18-23}$ However, without an initial perturbation, it is difficult for tearing modes alone to produce sufficiently large islands, and fast reconnection simply does not occur. Conceivably, the tiny islands could coalesce into larger islands until they reach the critical size necessary for fast reconnection physics. However, this mechanism is quite slow, and the present paper seeks other mechanisms that occur on much faster time scales. Finally, it is worth mentioning that the tearing instability is also believed to be suppressed by the presence of a relatively small vertical magnetic field (i.e., orthogonal to the current sheet), ${ }^{24-28}$ which makes it of questionable relevance in the magnetotail.

Our study of the onset of collisionless magnetic reconnection in a GEM challenge Harris sheet equilibrium ${ }^{13}$ without an initial perturbation shows that the lower-hybrid drift instability (LHDI) (see Ref. 29 for a recent review of the literature) has an important role in the onset of reconnection in three dimensions. In two dimensions, tearing instabilities saturate at low amplitudes, as expected. In three dimensions, the LHDI modifies the current sheet in ways that cause the 
tearing mode to grow more vigorously, as also observed by Sholer et al., ${ }^{30}$ and by Shinohara and Fujimoto. ${ }^{31}$

Waves at the lower hybrid frequency are observed near reconnection sites, ${ }^{32-34}$ and are often considered as a source of anomalous resistivity. In the earliest model of reconnection presented by Sweet and Parker, based on resistive MHD, the classical Spitzer resistivity is not able to explain the reconnection rates observed. Microinstabilities can enhance the plasma resistivity beyond the classical values, and thus, the LHDI, which is unstable to a broad range of wavelengths and frequencies, has been studied extensively as a source of anomalous resistivity. The fastest growing modes have wavelengths $k_{y} \rho_{e} \approx 1, \gamma \approx \Omega_{l h}$, are active in the low- $\beta$ region of the current sheet and are predominantly electrostatic. ${ }^{35}$ Longer wavelength modes $\left(k_{y} \sqrt{\rho_{i} \rho_{e}} \approx 1, \gamma \approx \Omega_{c i}\right)$ have a large electromagnetic component and for sufficiently thin sheets can penetrate into the center of the current sheet. ${ }^{36,37}$ However, observations show that the LHDI is confined to the edge of current sheets, its amplitude seems uncorrelated with the reconnection electric field, and it saturates at too low amplitude to explain the enhanced reconnection rate through an anomalous resistivity. ${ }^{32-34}$

Recently, the effects of the LHDI have been revisited. It has been observed that in the nonlinear phase of the evolution of the LHDI in a Harris sheet, modifications of the profiles are induced. First, the current sheet is thinned and the electron current profile is peaked. ${ }^{38-42}$ The effect of electron current sheet peaking is primarily due to electron acceleration and not due to electron density modification. ${ }^{30}$ From these results the possibility emerges that the modifications of the current profile induced by the LHDI allow the onset of secondary instabilities and modify the growth rates of instabilities already present in the initial equilibrium. Second, previous results based on two-dimensional simulations in the plane of the LHDI (orthogonal to the magnetic field and to the plane where tearing develops) have indeed shown that a Kelvin-Helmholtz instability (KHI) develops at much faster rates than predicted by linear theories based on the initial equilibrium (see Ref. 41 and references therein). Furthermore, previous three-dimensional simulations ${ }^{30,31,42,43}$ have suggested that the modification of the initial profile caused by the LHDI can allow the onset of reconnection and enhance the growth of the tearing instability.

In the present paper we revisit the issue with more detailed simulations and with more extensive diagnostics, by which we uncover new physics and confirm previous results. First, we discover that the nonlinear evolution of the LHDI not only heats electrons (an effect documented in many previous works, e.g., Ref. 36) but preferentially heats electrons perpendicular to the magnetic field. Anisotropic heating has a great impact on the onset of reconnection. Previous work $^{44-47}$ suggests that systems with an electron (or an ion) anisotropy are more unstable to the tearing instability when the perpendicular temperature exceeds the parallel temperature. We conduct a number of simulations and use linear Vlasov theory to calculate the effect of temperature anisotropy on tearing onset. Second, we confirm that the current sheet is thinned by the LHDI. The thinning can be explained by nonlinear effects of the LHDI. ${ }^{48}$ The thinning has the direct effect of promoting the growth of the tearing instability. We conduct a number of simulations and theoretical studies based on linear theory to estimate the effects of current sheet thinning on the linear growth rate.

In Sec. II, we describe our physical system and numerical approach. In Sec. III, we present our simulation results. In Sec. IV, we discuss our results and conclusions.

\section{PHYSICAL SYSTEM AND THE NUMERICAL APPROACH}

A Harris current sheet equilibrium is considered, ${ }^{12}$ with an initial magnetic field given by

$$
\mathbf{B}_{0}(z)=B_{0} \tanh (z / L) \mathbf{e}_{x}
$$

and a plasma density given by

$$
n_{0}(z)=n_{0} \operatorname{sech}^{2}(z / L) \text {. }
$$

In the present paper, similar parameters to the GEM challenge are used. ${ }^{13}$ In the GEM challenge, the current sheet thickness is $L=0.5 d_{i}$, the temperature ratio is $T_{i} / T_{e}=5$, and the ion drift velocity in the $y$ direction is $V_{i 0}=1.67 V_{A}$. Rather than the standard mass ratio, $m_{i} / m_{e}=180$ is used. The Alfvén velocity $V_{A}$ and the ion inertial length, $d_{i}=c / \omega_{p i}$, are defined with the density $n_{0}$ and the field $B_{0}$. In contrast to the GEM challenge, no background density is introduced. This is an important point, since the presence of a background is strongly stabilizing to the LHDI. The standard dimensionless parameters necessary to characterize a Harris current sheet can thus be summarized as, $\omega_{p e} / \Omega_{c e}=2.88, \rho_{i} / L=1.828$, $m_{i} / m_{e}=180$, and $T_{i} / T_{e}=5$, with $v_{t h, s} \equiv \sqrt{2 T_{s} / m_{s}}$. The boundary conditions for the particles and fields are periodic in the $x$ and $y$ directions. Conducting boundary conditions are imposed for the fields at the $z$ boundaries while reflecting boundary conditions are used for the particles. In order to study the reconnection onset and in contrast to the GEM challenge, ${ }^{13}$ the Harris equilibrium is initially unperturbed.

We simulate the dynamics in both the reconnection, $(x, z)$, and current-aligned, $(y, z)$, planes. In the $(x, z)$ plane, the domain is $L_{x} \times L_{z}=25.6 \mathrm{~L} \times 12.8 \mathrm{~L}$, corresponding to $L_{x}$ $\times L_{z}=12.8 d_{i} \times 6.4 d_{i}$. (The GEM challenge domain is $L_{x}$ $\times L_{z}=51.2 L \times 25.6 L$. $)$ In the $(y, z)$ plane, the domain is $L_{y}$ $\times L_{z}=32 L \times 12.8 L$. We also perform a three-dimensional simulation, for which the domain is $L_{x} \times L_{y} \times L_{z}=25.6 L$ $\times 16 L \times 12.8 L$. (This simulation is performed with CELESTE3D only, which is described below.)

To investigate the evolution of the system, a linear Vlasov code and two different nonlinear particle-in-cell (PIC) simulation codes are used. The linear Vlasov results for the tearing mode are calculated using the formally exact approach described in Refs. 37 and 49. This technique employs a normal mode calculation using a full Vlasov description for both ions and electrons. The orbit integrals arising from the linear Vlasov theory are treated numerically using the exact unperturbed particle orbits and including the form of the perturbation inside the integral. Both electromagnetic and electrostatic contributions to the field perturbation are retained and resulting system of integrodifferential equations is 
TABLE I. Simulation parameters.

\begin{tabular}{lccc}
\hline \hline & Grid & Particles & $\Delta t \Omega_{c e}$ \\
\hline$(x, z)$ plane (NPIC) & $1280 \times 640$ & $16 \times 10^{7}$ & 0.03 \\
$(x, z)$ plane (CELESTE3D) & $64 \times 64$ & $5 \times 10^{5}$ & 0.45 \\
$(y, z)$ plane (NPIC) & $1600 \times 640$ & $2 \times 10^{8}$ & 0.03 \\
$(y, z)$ plane (CELESTE3D) & $128 \times 64$ & $1 \times 10^{5}$ & 0.7 \\
$(x, y, z)$ (CELESTE3D) & $32 \times 64 \times 32$ & $3.5 \times 10^{6}$ & 0.9 \\
\hline \hline
\end{tabular}

solved using a finite element expansion of the eigenfunction. ${ }^{37}$ The basic strategy involves a normal mode calculation for perturbations of the form

$$
\begin{aligned}
& \hat{\phi}=\widetilde{\phi}(z) \exp \left(-i \omega t+i k_{y} y+i k_{x} x\right), \\
& \hat{\mathbf{A}}=\tilde{\mathbf{A}}(z) \exp \left(-i \omega t+i k_{y} y+i k_{x} x\right),
\end{aligned}
$$

where the complex functions $\hat{\boldsymbol{\phi}}, \hat{\mathbf{A}}$ are the perturbed electrostatic and vector potentials. For a given Vlasov equilibrium and for a given choice of wave vector $\left(k_{x}, k_{y}\right)$, the code computes the real frequency, growth rate (real and imaginary part of $\omega$ ), and the complex eigenfunctions $\widetilde{\phi}(z)$ and $\widetilde{\mathbf{A}}(z)$ which describe the mode structure.

The nonlinear dynamics are simulated by two PIC codes, an explicit simulation code NPIC, and an implicit simulation code CELESTE3D. The explicit plasma simulation code NPIC is based on a well-known explicit electromagnetic algorithm. ${ }^{50,51}$ The particle trajectories within NPIC are advanced using the leapfrog technique, and particle moments are accumulated with area weighting. The simulations are run on a parallel computer, using domain decomposition with calls to the message passing interface library. (In the present work, the explicit simulations are run on the Los Alamos Q-machine using as many as 128 nodes.) The implicit plasma simulation code, CELESTE3D, solves the full set of Maxwell-Vlasov equations using the implicit moment method. ${ }^{52-54}$ Both Maxwell's and Newton's equations are discretized implicitly in time. The implicit simulations are run on a workstation.

The nonlinear simulations are performed by the two codes with very different simulation parameters. In the tearing plane, NPIC employs a $N_{x} \times N_{z}=1280 \times 640$ grid, a time step $\Omega_{c e} \Delta t=0.03$, and $160 \times 10^{6}$ particles. CELESTE3D uses a $N_{x} \times N_{z}=64 \times 64$ grid, with time step $\Omega_{c e} \Delta t=0.45$, and a total of $5 \times 10^{5}$ computational particles. In the current aligned plane, NPIC employs $N_{y} \times N_{z}=1600 \times 640$ grid, a time step $\Omega_{c e} \Delta t=0.03$, and $2 \times 10^{8}$ particles, while CELESTE3D uses a $N_{x} \times N_{z}=128 \times 64$ grid, with time step $\Omega_{c e} \Delta t=0.7$, and a total of $1 \times 10^{5}$ computational particles. The three-dimensional simulation performed by CELESTE3D employs a grid $N_{x} \times N_{y} \times N_{z}=32 \times 64 \times 32$, a time step $\Omega_{c e} \Delta t=0.9$ and $3.5 \times 10^{6}$ computational particles. The simulation parameters are summarized in Table I.

Detailed comparison of the plasma dynamics in NPIC and CELESTE3D are made in a study of the nonlinear phase of magnetic reconnection in plasmas with different $\beta$ values. $^{23}$ The comparison shows that the physical mecha- nisms revealed by the two codes agree, which increases confidence in their validity. Similar comparisons are made with other codes for the GEM challenge, which show that results with CELESTE3D are comparable in detail with those of explicit simulations. ${ }^{54,55}$ The same kinds of comparisons are made in this study.

\section{SIMULATION RESULTS}

To study the onset of reconnection, we simulate the reference configuration defined above, similar to the GEM challenge, ${ }^{13}$ but without initial perturbation, without background plasma, $m_{i} / m_{e}=180$, and with a smaller box size. It is left to the natural noise of PIC to excite any instability. The result is strikingly different from published GEM challenge results. The tearing mode saturates at low amplitude, and the reconnected flux is a small fraction of that available. If a third dimension in the current aligned direction, $y$, is added to the simulation of the same physical system, the tearing mode grows to much larger amplitude before saturating and reconnection occurs. Two effects of the LHDI instability appear to cause a dramatic change in both the linear and the nonlinear phase of the tearing instability in threedimensional dynamics, compared with the two-dimensional dynamics. They are anisotropic electron heating and current sheet thinning. Both of these effects enhance the linear growth rate of the tearing instability and strongly increase the saturation amplitude. In the following sections, the results of the simulations are described in detail.

\section{A. Linear tearing and saturation \\ 1. Two-dimensional simulation}

We consider a two-dimensional simulation in the tearing plane $(x, z)$, with the parameters defined above, similar to the GEM challenge. For this configuration, linear theory predicts that the fastest growing tearing mode has wavelength $k_{x} L$ $=0.5$ and growth rate $\gamma=0.176 \Omega_{c i}$. In Fig. 1, the amplitude of tearing modes with $m_{x}=1,2,3$, and 4 , corresponding to $k_{x} L=0.25,0.5,0.75$, and 1 , are shown as a function of time. The growth of higher mode numbers is negligible. At the beginning of the simulation, Fig. 1 shows the fast growth of the tearing mode with $m_{x}=1$ and $m_{x}=2$. The comparison between linear theory and the actual growth rate measured from the PIC simulation is shown in Table II. The comparison shows a good agreement in the first period of the simulation (the small differences can be due to nonlinear coupling between modes and noise from PIC simulation); on longer time scales, the PIC simulation indicates that the growth rates decrease. The dominance of the mode with $m_{x}=1$ reveals the presence of a single magnetic island that involves the whole domain.

The tearing instability saturates at a low level. The half width of the island at saturation is $w \approx 0.46 \mathrm{~L}$ at time $t \Omega_{c i}$ $=83$. By comparison, GEM challenge reconnection encompasses the whole domain at $t \Omega_{c i} \approx 30 \quad(w \approx 10 L)$. CELESTE3D and NPIC agree in showing low amplitude saturation of the tearing mode, but the coarser grid spacing used in CELESTE3D does not allow an accurate estimate of the island half width. It should be noted that the growth of 


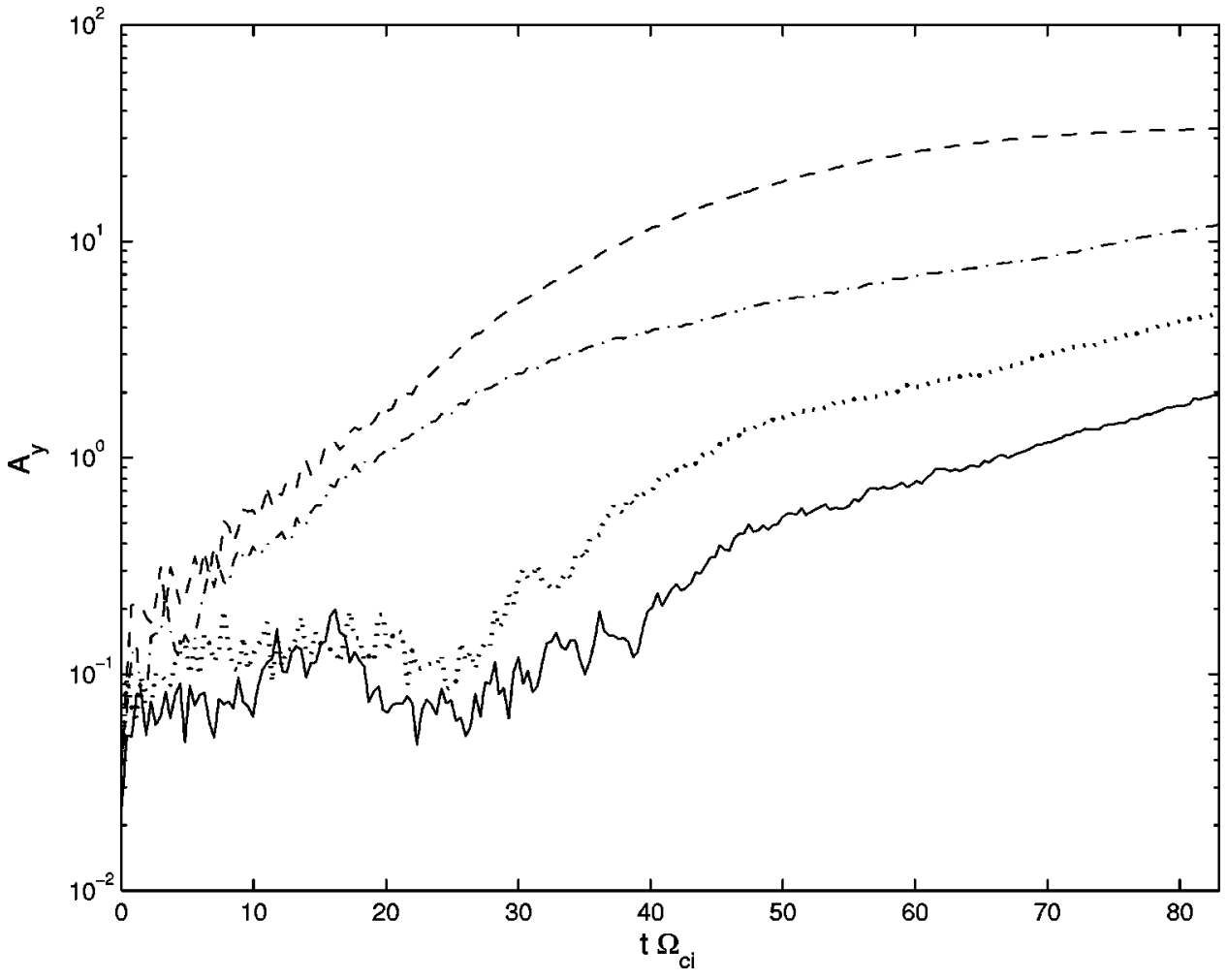

FIG. 1. The growth of the tearing mode is shown for the modes $m_{x}=1$, $k_{x} L=0.25$ (dashed line), $m_{x}=2, k_{x} L$ $=0.5$ (dash-dotted line), $m_{x}=3, k_{x} L$ $=0.75$ (dotted line), and $m_{x}=4, k_{x} L$ $=1$ (solid line) from two-dimensional simulations with NPIC in the tearing, $(x, z)$, plane. tearing modes in NPIC with $m_{x}=3,4$ at $t \Omega_{c i} \approx 80$ is possibly due to the numerical heating always present in explicit PIC simulations like NPIC, which can in principle affect the results of long time scale simulations. In any case, this slow growth at late time in the two-dimensional tearing simulation is several orders of magnitude slower than the growth and nonlinear development of the LHDI in three dimensions that is the subject of the present work.

The saturation of tearing has been studied theoretically in high- and low- $\beta$ plasmas (e.g., see Refs. 17, 56, and 57). In high- $\beta$ plasmas, like the ones considered in the GEM challenge, anisotropic heating of electrons causes saturation of tearing. ${ }^{17}$ During the growth of the tearing instability, $\left(T_{e y y}+T_{e z z}\right) / 2 T_{e x x}$ (defined as $\left.T_{e \perp} / T_{e \|}\right)$ is reduced below 1 , and $T_{e \perp} / T_{e \|}<1$ has a strongly stabilizing effect on tearing. In fact, in the two-dimensional simulations of tearing at time $t \Omega_{c i}=80$, NPIC gives $T_{e \perp} / T_{e \|}=0.87$ and CELESTE3D gives $T_{e \perp} / T_{e \|}=0.83$. (The temperatures are averaged over $x$ in the interval $-0.2 L \leqslant z \leqslant 0.2 L$ for NPIC and $-0.3 L \leqslant z \leqslant 0.3 L$ for CELESTE3D.) For $T_{e \perp} / T_{e \|}=0.87$ the linear code predicts the mode $m_{x}=2\left(k_{x} L=0.5\right)$ to be stable and the mode $m_{x}$ $=1\left(k_{x} L=0.25\right)$ to be weakly unstable $\left(\gamma=0.04 \Omega_{c i}\right)$. However,

TABLE II. Growth rate of the tearing instability from the linear theory and from the least square regression of the two-dimensional NPIC simulation results in the specified period.

\begin{tabular}{cccc}
\hline \hline Mode $m_{x}$ & Linear theory & NPIC $\left(0-10 / \Omega_{c i}\right)$ & NPIC $\left(0-20 / \Omega_{c i}\right)$ \\
\hline 1 & 0.136 & 0.14 & 0.11 \\
2 & 0.176 & 0.16 & 0.12 \\
\hline \hline
\end{tabular}

this growth rate is based on a bi-Maxwellian electron distribution. The simulated velocity distribution is more complex and might yield a different growth rate.

It should be remarked that in simulations performed in a more extended domain, small islands can coalesce repeatedly into larger islands. ${ }^{58}$ If the system is big enough, the final merged island can grow enough to allow the fast reconnection physics shown by the GEM challenge. However, this coalescence process occurs on a long time scale compared to the time scales typical of the three-dimensional instabilities that are the subject of the present manuscript.

\section{Three-dimensional simulation}

CELESTE3D is used to perform a three-dimensional simulation of the Harris current sheet described in Sec. II, in a domain with $L_{x} \times L_{y} \times L_{z}=25.6 L \times 16 L \times 12.8 L$. Tearing, current aligned (i.e., LHDI and KHI), and oblique instabilities are all involved in the evolution of the system. In previous work, the results of three-dimensional simulations performed by CELESTE3D have been directly compared with satellite observations. ${ }^{59}$ A close agreement has been observed between simulation and satellite observations, concerning current sheet kinking, current bifurcation, and reconnecting modes.

In Fig. 2, the amplitude of the Fourier component of the $B_{x}$ magnetic field is plotted as a function of the mode number $m_{x}$ (aligned with the magnetic field) and $m_{y}$ (aligned with the current) at sequential times. The plots average over $z$ and fast Fourier transform in $(x, y)$. The average over $z$ suppresses odd parity modes in $B_{x}$ and thus only even parity modes, such as KHI and LHDI, appear in Fig. 2. Since $B_{x}$ and $B_{z}$ 
a)
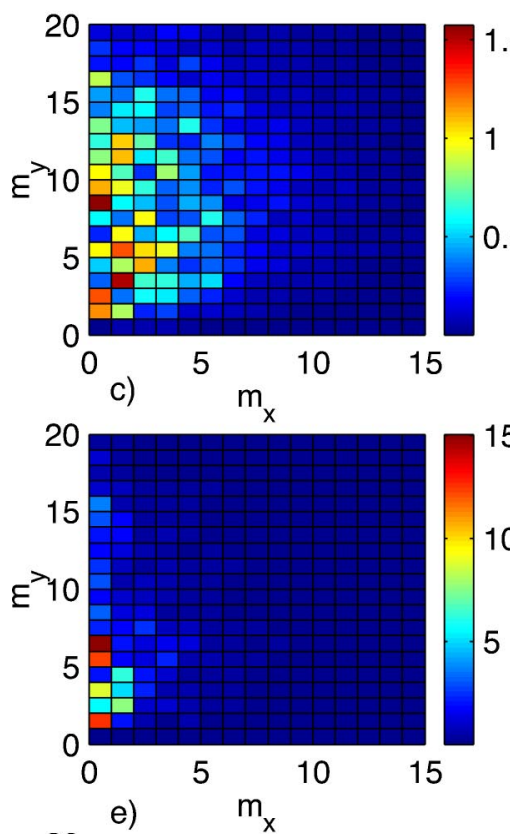

b)

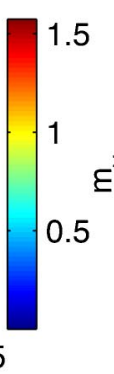

.5

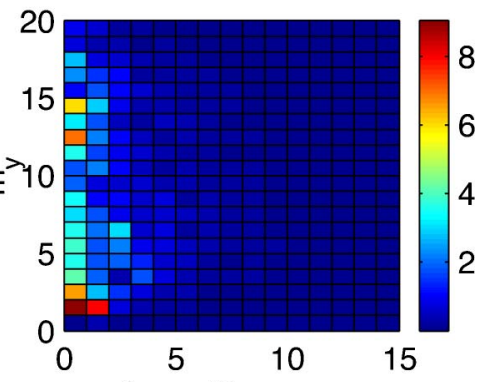

d) $\quad m_{x}$

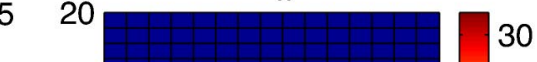

15
20 $\varepsilon$

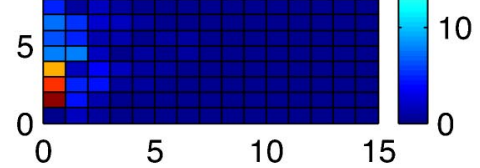

f) $\quad m_{x}$

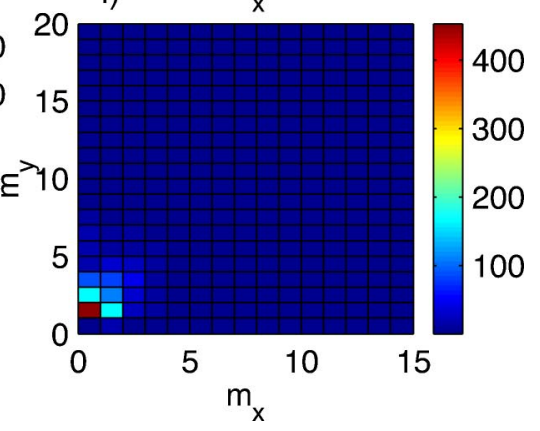

FIG. 2. (Color online). The amplitude of the Fourier components of $B_{x}$ from a three-dimensional simulation with CELESTE3D are shown as a function of the mode numbers $m_{x}$ and $m_{y}$ at $t \Omega_{c i}=0.2$ (a), $t \Omega_{c i}=2.5(\mathrm{~b}), t \Omega_{c i}$ $=5(\mathrm{c}), t \Omega_{c i}=10(\mathrm{~d}), t \Omega_{c i}=15(\mathrm{e})$, and $t \Omega_{c i}=20$ (f). have opposite parity, the even modes, such as the tearing modes (not shown in Fig. 2), are picked up by $z$ averages of Fourier modes of $B_{z}$.

Figure 2 shows the development of the initial noise, which excites mostly low mode number instabilities $m_{x}$ and $m_{y}$ at $t \Omega_{c i}=0.2$. The presence of the electrostatic LHDI is reflected at time $t \Omega_{c i}=2.5$ through a significant amplitude of the mode number $m_{x}=0, m_{y}=12-14$, which corresponds to $k_{y} \rho_{e} \approx 0.2$. The electrostatic LHDI, studies of which are summarized in Ref. 29, is dominated by an electrostatic component on the edge of the current sheet. The characteristic wavelength is of order $k_{y} \rho_{e} \approx 1$ and growth rate $\gamma \approx \Omega_{l h}{ }^{35}$ The electrostatic LHDI instability causes a velocity shear that enhances the growth rate of the electromagnetic LHDI and causes a KHI to grow. ${ }^{40,41}$ Later, at $t \Omega_{c i}=5$, the maximum amplitude modes are $m_{x}=0$ and $m_{y}=5-6$, which corresponds to $k_{y} \sqrt{\rho_{i} \rho_{e}} \approx 0.5$. These longer wavelength modes are the electromagnetic LHDI, which grows at center of the current sheet and has wavelength $k_{y} \sqrt{\rho_{i} \rho_{e}} \approx 1$ and growth rate $\gamma \approx \Omega_{c i}{ }^{37}$ Velocity shear created by the LHDI triggers a KHI at a still later time, so that by $t \Omega_{c i}=20$ the current sheet is dominated by a single, domain-sized kink with mode numbers $m_{x}=0$ and $m_{y}=1\left(k_{y} L \approx 0.4\right)$.

Figure 3 shows the amplitude of the Fourier modes of the $B_{z}$ field as a function of $m_{x}$ and $m_{y}$ at different times. Again, averaging over $z$ eliminates the odd parity modes and leaves only even parity modes. The modes shown in Fig. 3 are complementary to the those in Fig. 2. The $B_{z}$ Fourier components are signatures of the tearing instability. The tearing instability starts to grow significantly at $t \Omega_{c i}=5$, with mode number $m_{x}=4$ and $m_{y}=0\left(k_{x} L=1\right.$, a shorter wavelength than expected from the linear theory based on the initial Harris equilibrium). Subsequently, the four magnetic islands merge into two islands at $t \Omega_{c i}=10$, and then into one that encompasses the whole domain by $t \Omega_{c i} \approx 20$. This is comparable to the reconnection time shown by the GEM challenge simulation in two dimensions with a large initial perturbation. $^{13}$ No significant growth of oblique tearing modes is observed during the simulation.

Two main conclusions can be drawn from the threedimensional simulation. First, in contrast to simulations in two dimensions, the tearing mode does not saturate at a low amplitude in three dimensions. Instead, it encompasses the whole domain in a time comparable to the GEM challenge simulation with a large initial perturbation. ${ }^{13}$ Second, no significant growth of oblique modes is seen in the simulation.

Thus, it can be argued that the current sheet dynamics depends on some nonlinear interaction between the tearing instability, which develops in the $(x, z)$ plane, and current aligned instabilities, which grow in the $(y, z)$ plane. As the time scale of instabilities in the $(y, z)$ plane is an order of magnitude faster than the tearing instability, it follows that 
a)
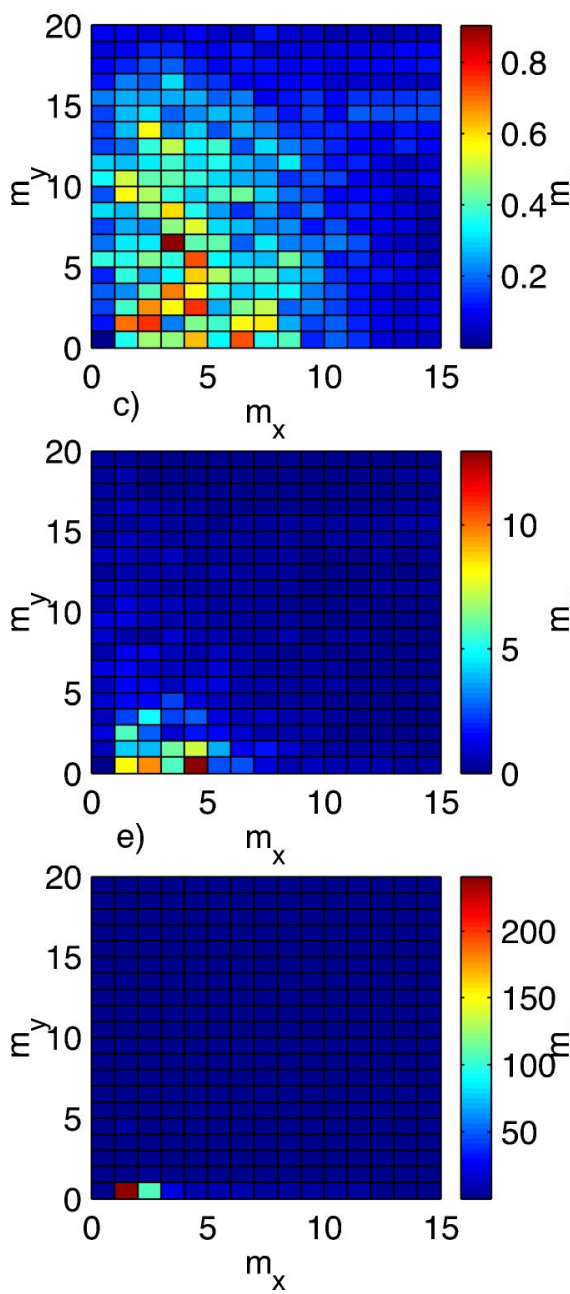

b)

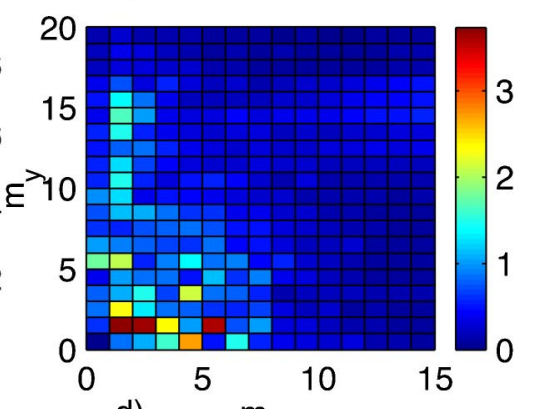

d) $m_{x}$

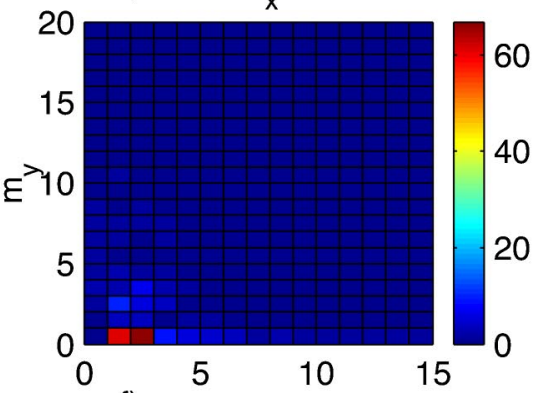

f) $m_{x}$

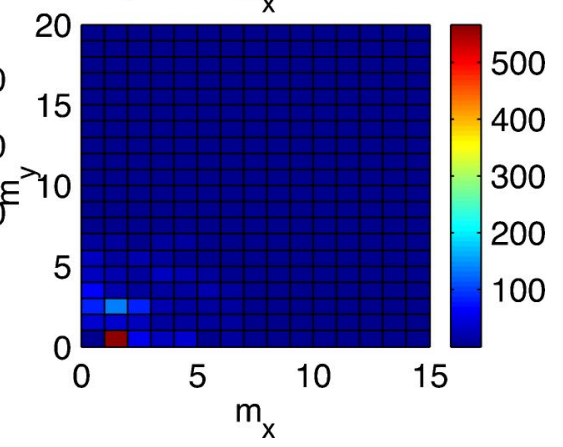

FIG. 3. (Color online). The amplitude of the Fourier components of $B_{z}$ from three-dimensional simulations with CELESTE3D are shown as a function of the mode numbers $m_{x}$ and $m_{y}$ at $t \Omega_{c i}=0.2$ (a), $t \Omega_{c i}=2.5$ (b), $t \Omega_{c i}=5$ (c), $t \Omega_{c i}=10(\mathrm{~d}), t \Omega_{c i}=15(\mathrm{e})$, and $t \Omega_{c i}$ $=20$ (f). the fundamental dynamics of a three-dimensional current sheet can be understood more conveniently by performing simulations in the $(x, z)$ and $(y, z)$ plane, and analyzing the effects of the very rapid current aligned instability on the more slowly growing tearing mode.

\section{B. Current sheet modifications by the LHDI}

In simulations performed in the current aligned $(y, z)$ plane, two modifications of the initial equilibrium appear to enhance the tearing growth rate. Both are nonlinear consequences of the LHDI.

The LHDI causes anisotropic heating of the electrons. In Fig. 4 , the $y$-averaged electron temperature ratio $T_{e \perp} / T_{e \|}$ is plotted as a function of $z$ at $t \Omega_{c i}=0$ and at $t \Omega_{c i}=5$. In both the CELESTE3D and NPIC simulations, an electron temperature anisotropy develops by $t \Omega_{c i}=5$ both at the center and on the flanks of the current sheet. The anisotropy ranges between 2.3 in NPIC and 1.7 in CELESTE3D. The ratio between the ion perpendicular and parallel temperature, $T_{i \perp} / T_{i \|}$, at $t \Omega_{c i}=0$ and at $t \Omega_{c i}=5$, is also shown in Fig. 4. According to both PIC codes, ion anisotropic heating is smaller than electron heating, and it is located mostly on the flanks of the current sheet. As shown in Fig. 5, the electron distribution function is not gyrotropic. In particular, the peak electron perpendicular temperature in the $z$ direction (cross sheet direction) is about $25 \%$ higher than the temperature in the $y$ direction (current aligned direction). Moreover, the heating in the $z$ direction is more focused near the center of the current sheet than the heating in the $y$ direction.

The LHDI also alters the current profile. It thins the current sheet and increases the value of the peak current density. The alteration is primarily due to electron acceleration: the ion and the electron density modifications are small. $^{30,31,38,41,42}$ In Fig. 6, the $y$-averaged current sheet profile is shown at $t=0$ and at $t \Omega_{c i}=4$ as a function of $z$. The current profiles obtained from both PIC codes agree well and show that the peak current is enhanced by about $40 \%$ over the initial current profile.

The LHDI also creates velocity shear. This latter consequence has been the subject of a number of papers (see Ref. 41 and references therein). Its main effect is to promote the growth of a KHI, but the three-dimensional simulation shows that the KHI develops after the onset of reconnection and thus appears to have little effect on reconnection onset.

\section{Effects of the LHDI on tearing}

Both anisotropic heating and current peaking enhance the linear growth rate of the tearing instability and change 

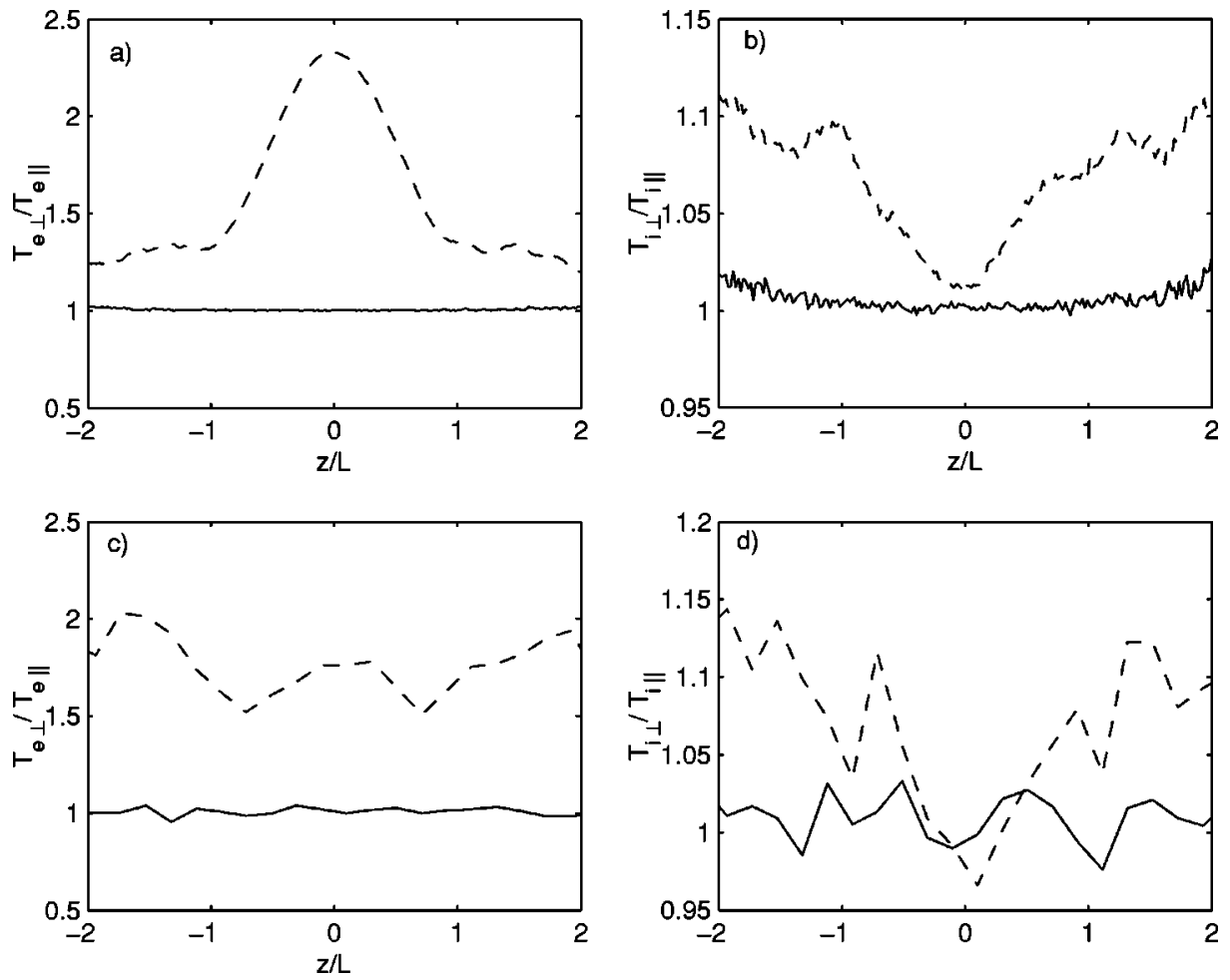

FIG. 4. The enhancement of the electron and ion temperature ratios $T_{e \perp} / T_{e \|}(\mathrm{a}, \mathrm{c})$, and $T_{i \perp} / T_{i \|}(\mathrm{b}, \mathrm{d})$ by the LHDI is shown from simulations in the $(y, z)$ plane. The temperature ratio is shown at time $t=0$ (solid line, isotropic distribution) and $t \Omega_{c i}=5$ (dashed line). The results are from NPIC $(a, b)$ and CELESTE3D (c,d) simulations. dramatically its nonlinear evolution. The effects of the two mechanisms on tearing are considered independently in the following sections.

\section{Electron anisotropy}

Figure 7 shows the value of the tearing growth rate $\gamma$ as a function of $k_{x}$ for $T_{e \perp} / T_{e \|}=0.9,1,1.5,2$, with the GEM challenge parameters (in particular $T_{i} / T_{e \|}=5$ ). The growth rate of the tearing instability increases appreciably with the ratio $T_{e \perp} / T_{e \|}$ : for $T_{e \perp} / T_{e \|}=2$ the maximum growth rate, $\gamma$ $\approx 5.1 \Omega_{c i}$, is more than one order of magnitude larger than the maximum growth rate for $T_{e \perp} / T_{e \|}=1 \quad\left(\gamma \approx 0.18 \Omega_{c i}\right)$. Moreover, the tearing mode with maximum growth rate shifts to shorter wavelengths with increasing anisotropy: in the case $T_{e \perp} / T_{e \|}=2$ the maximum occurs for $k_{x} L=2.25$, while typically $k_{x} L=0.5$ for isotropic electrons. It should be remarked

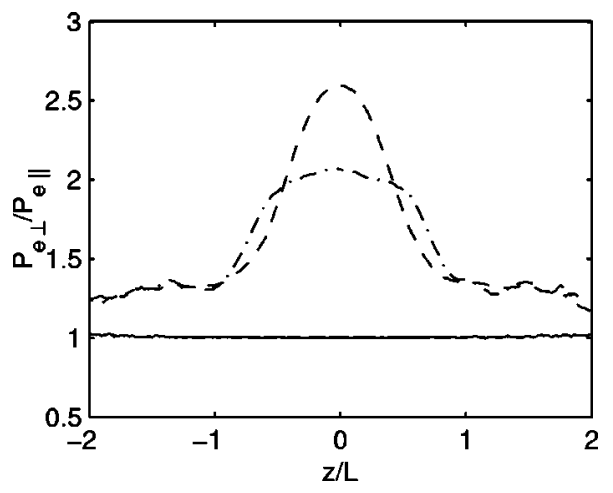

FIG. 5. The electron pressure ratios $P_{e z z} / P_{e x x}$ (dashed line) and $P_{e y y} / P_{e x x}$ (dash-dotted line) are averaged along $y$ and plotted at time $t \Omega_{c i}=5$. The values for $P_{e z z} / P_{e x x}$ (solid line) and $P_{e z z} / P_{e x x}$ (dotted line) at $t=0$ are shown for comparison. The results are from an NPIC simulation in the $(y, z)$ plane. that electron heating due to LHDI does not lead to a simple bi-Maxwellian electron distribution function. In Fig. 5, the difference between the pressure terms $P_{e y y}$ and $P_{e z z}$ resulting from the NPIC simulation are shown. Nevertheless, we assume a bi-Maxwellian distribution function to roughly estimate the growth rate of the tearing instability.

The tearing eigenmodes for $T_{e \perp} / T_{e \|}=2$ and $T_{e \perp} / T_{e \|}=1$ are compared in Fig. 8. The most remarkable difference is in the structure of the potential $\phi$, which is larger in the presence of anisotropic electrons and with opposite sign. Moreover, for anisotropic electrons, the tearing eigenmode involves a narrower region near the center of the current sheet and the $A_{z}$ component is more important.

These results are consistent with the enhancement of the tearing instability growth rates predicted in previous work, ${ }^{44-47,60-62}$ but our results are obtained by integration over exact orbits, similarly to Ref. 38. As an aside, it should be remarked that our linear analysis predicts that ion anisotropy plays only a minor role in thin current sheets $\left(\rho_{i} \sim L\right)$ in agreement with earlier work by Burkhart and Chen. ${ }^{38}$

The nonlinear evolution of the tearing mode changes dramatically when the electron temperature is anisotropic. An initially favorable temperature anisotropy neutralizes the effect of the unfavorable anisotropy created by the growth of tearing, which would otherwise cause nonlinear stabilization. The evolution of the current sheet is followed in twodimensional PIC simulations in the $(x, z)$ plane, starting with an anisotropic electron temperature. The GEM challenge parameters are used with $T_{i} / T_{e \|}=5$ on the same domain $L_{x}$ $\times L_{z}=25.6 L \times 12.8 L$, but with various electron temperature anisotropies: $T_{e \perp} / T_{e \|}=1,1.5$, and 2 . In Fig. 9, the evolution of the tearing instabilities with mode numbers $m_{x}=1-11$ (corresponding to $k_{x} L=0.25-3$ ) are shown as a function of 


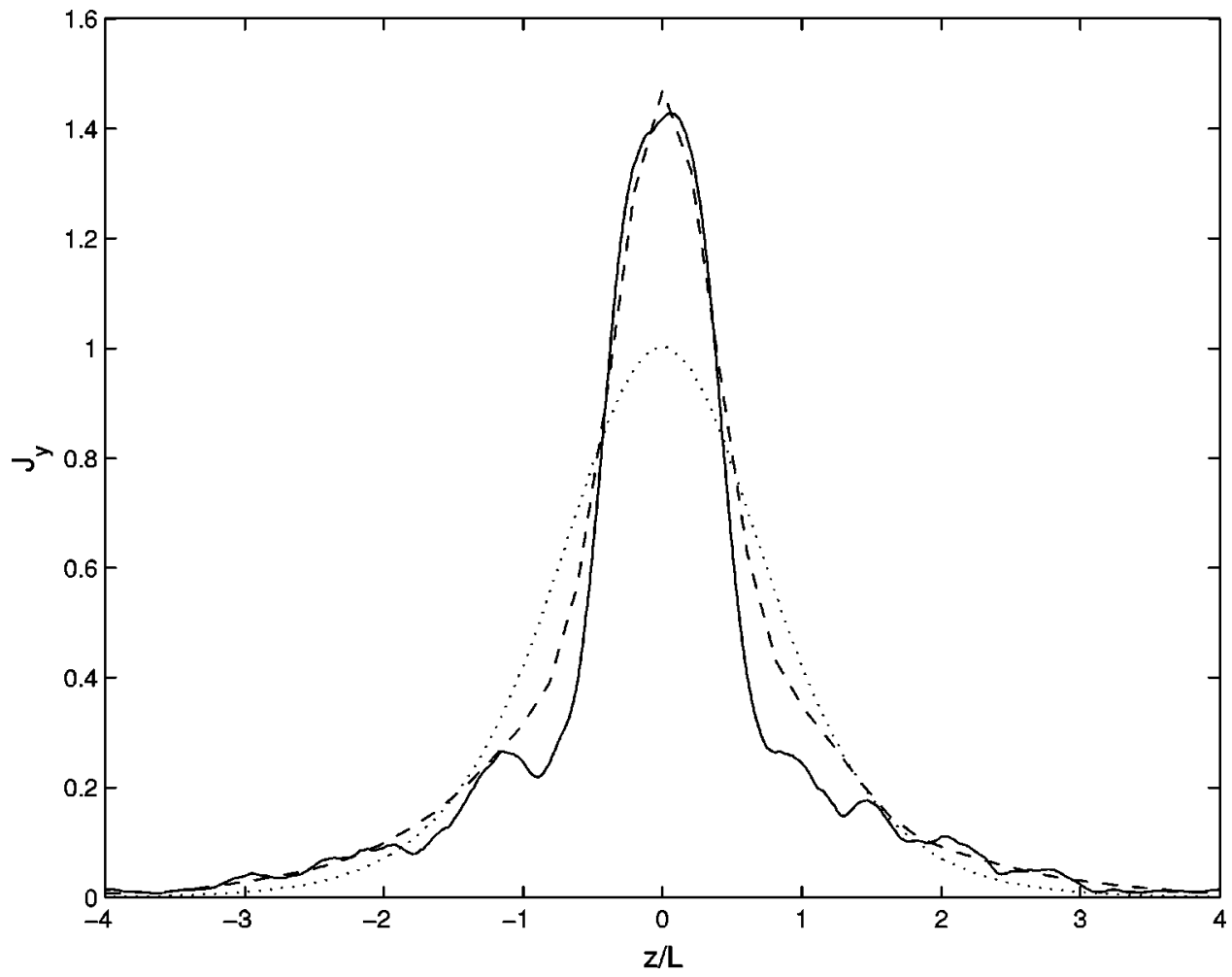

FIG. 6. The peaking of the current density $J_{y}$ is shown from simulations in the $(y, z)$ plane. The dotted line represents the current profile at $t=0$ averaged along $y$. Profiles at $t \Omega_{c i}=4$ are shown by a solid line (NPIC simulation) and a dashed line (CELESTE3D simulation). The current is normalized so that the maximum is equal to 1 at $t=0$.

time. In comparison with the case for initially isotropic electrons (see Fig. 1), high mode numbers grow, forming a number of small scale islands. Subsequently, these small islands merge to form a single large island (the mode number $m_{x}$ $=1$ dominates), which encompass the whole domain by $t \Omega_{c i} \approx 20$.
In Fig. 10, the reconnected flux is plotted as a function of time for the three different anisotropies, $T_{e \perp} / T_{e \|}=1,1.5$, and 2, for both NPIC and CELESTE3D simulations. With $T_{e \|} / T_{e \perp}=1$, tearing saturates at a low level. For anisotropic distributions, $T_{e \perp} / T_{e \|}=1.5,2$, reconnection involves the whole domain and the growth of the tearing instability satu-

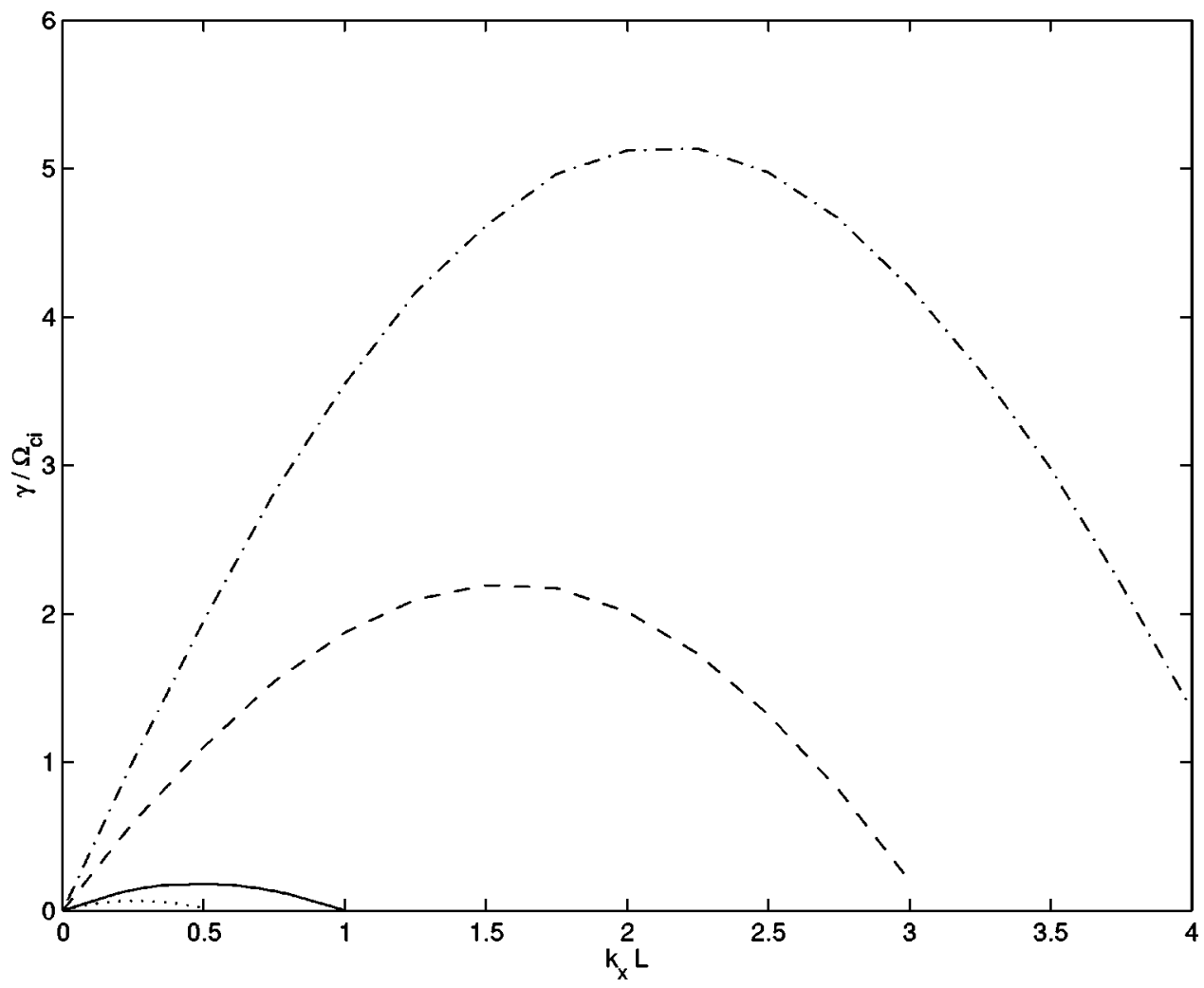

FIG. 7. The growth rate $\gamma / \Omega_{c i}$ of the tearing mode is plotted as a function of $k_{x} L$ for $T_{e \perp} / T_{e \|}=0.9$ (dotted), $T_{e \perp} / T_{e \|}=1 \quad$ (solid), $\quad T_{e \perp} / T_{e \|}=1.5$ (dashed), and $T_{e \perp} / T_{e \|}=2$ (dashdotted). The other plasma parameters are the same as the parameters described in Sec. II (in particular, $T_{i} / T_{e \|}=5$ ). 

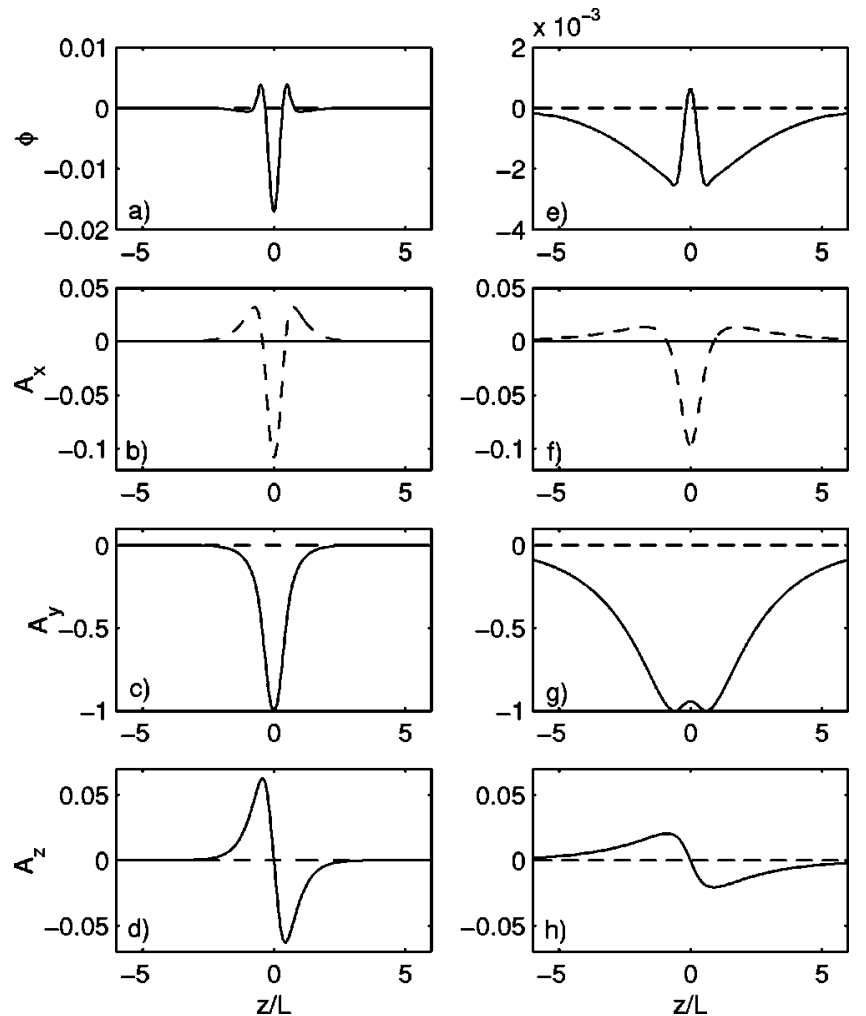

FIG. 8. The eigenmodes for the fastest growing tearing instability are shown. The real (solid) and imaginary (dotted) parts are plotted for $\phi(\mathrm{a}, \mathrm{e})$, $A_{x}(\mathrm{~b}, \mathrm{f}), A_{y}(\mathrm{c}, \mathrm{g})$, and $A_{z}(\mathrm{~d}, \mathrm{~h})$, with $T_{e \perp} / T_{e \|}=2, k_{x} L=2.25$, and $\gamma=5.13 \Omega_{c i}$ (a,b,c,d); and with $T_{e \perp} / T_{e \|}=1, k_{x} L=0.5$, and $\gamma=0.176 \Omega_{c i}(\mathrm{e}, \mathrm{f}, \mathrm{g}, \mathrm{h})$.

rates when all the available magnetic flux is reconnected, at a level very similar to the GEM challenge. The fast reconnection phase is delayed longer for $T_{e \perp} / T_{e \|}=1.5$ than for $T_{e \perp} / T_{e \|}=2$.

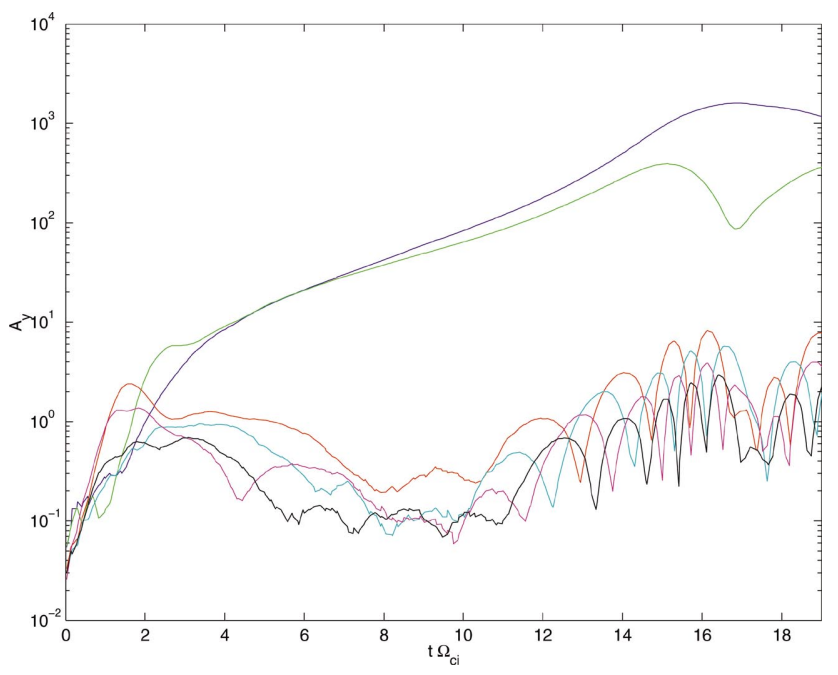

FIG. 9. (Color). The amplitude of the tearing mode is shown as a function of time for the long wavelength modes: $m_{x}=1$ and $k_{x} L=0.25$ (blue), $m_{x}=2$ and $k_{x} L=0.5$ (green), and for short wavelength modes: $m_{x}=9$ and $k_{x} L=2.25$ (red), $m_{x}=10$ and $k_{x} L=2.5$ (cyan), $m_{x}=11$ and $k_{x} L=2.75$ (magenta), and $m_{x}=12$ and $k_{x} L=3$ (black), from a two-dimensional simulation with NPIC in the tearing plane with plasma parameters described in Sec. II, but with $T_{e \perp} / T_{e \|}=2$ and $T_{i} / T_{e \|}=5$. a)
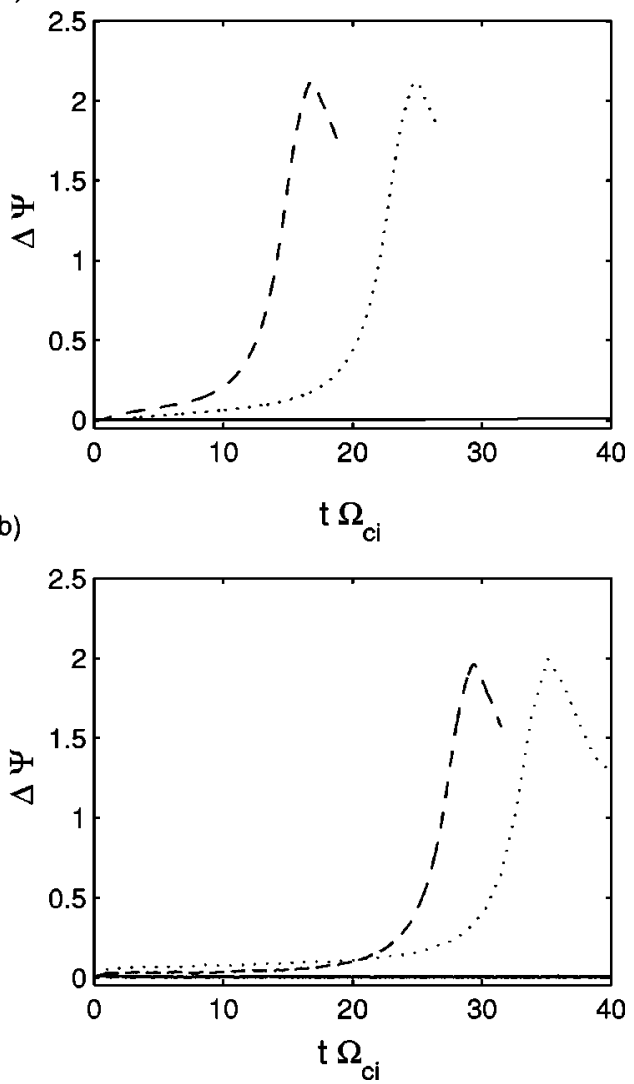

FIG. 10. The change in the reconnected flux $\Delta \Psi$ with time, is compared for NPIC (a) and CELESTE3D (b) simulations. The parameters are as described in Sec. II with $T_{i} / T_{e \|}=5$ and $T_{e \perp} / T_{e \|}=1$ (solid line), $T_{e \perp} / T_{e \|}=1.5$ (dotted line), and $T_{e \perp} / T_{e \|}=2$ (dashed line).

Figure 11 shows a plot of the temperature anisotropy as a function of time for the three simulations with different initial anisotropies $\left(T_{e \perp} / T_{e \|}=1,1.5,2\right)$. The temperature ratio $\left(T_{e y y}+T_{e z z}\right) / 2 T_{e x x}$ that corresponds to $T_{e \perp} / T_{e \|}$ at $t=0$ is plotted. For an initially isotropic distribution, $T_{e \perp} / T_{e \|}=1$, the ratio decreases as a function of time and stabilizes the tearing instability. For initially anisotropic distributions, the ratio $T_{e \perp} / T_{e \|}$ decreases rapidly to a minimum value, $T_{e \perp} / T_{e \|}$ $\approx 0.8$, and then increases with the onset of fast reconnection.

\section{Current thinning and peaking}

The thinning of the current sheet and consequent increase in maximum current density also enhances the linear growth rate of the tearing instability and changes its nonlinear evolution. This is in agreement with the results of other recent simulation studies. ${ }^{30,31}$

Figure 12 shows the tearing instability linear growth rate $\gamma$ for different values of the current sheet thickness $\left(\rho_{i} / L\right.$ $=0.914,1.828,3.656)$. The growth rate is higher for thinner current sheets: $\gamma=0.176 \Omega_{c i}$ for $\rho_{i} / L=1.828$ (GEM challenge thickness) compared with $\gamma=0.632 \Omega_{c i}$ for $\rho_{i} / L=3.656$. The maximum growth rate is located at $k_{x} L=0.5$ in all cases.

The nonlinear evolution of the tearing instability is simulated with both NPIC and CELESTE3D. For sufficiently thin current sheets, reconnection is not blocked by nonlinear saturation of the tearing mode at low levels and encompasses the 
a)

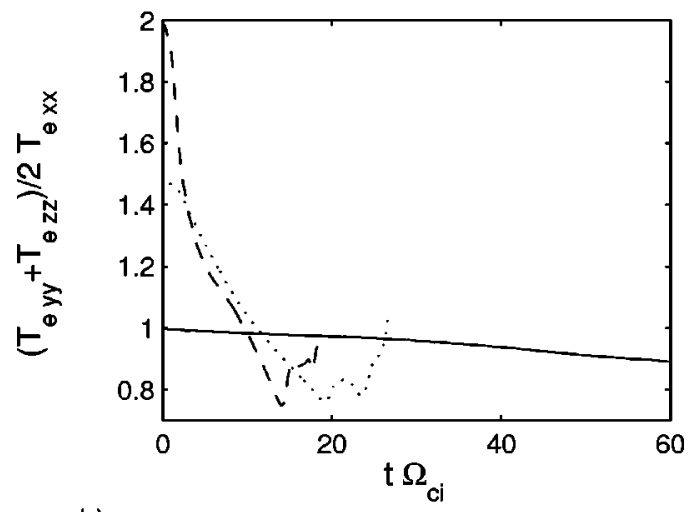

b)

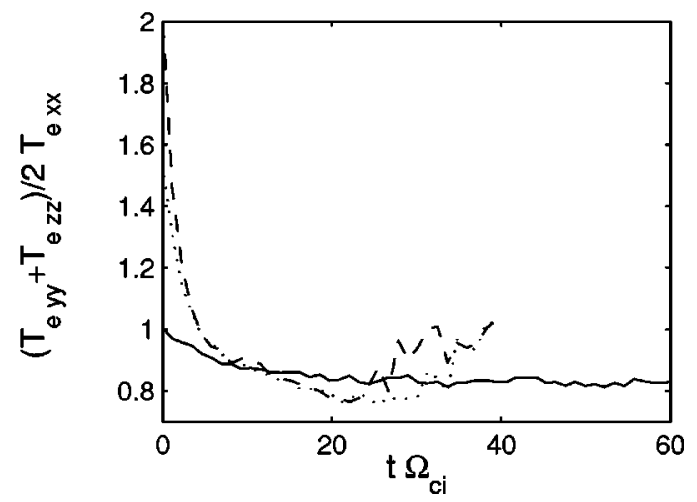

FIG. 11. The electron temperature ratio $\left(T_{e y y}+T_{e z z}\right) / 2 T_{e x x}$ (corresponding to $T_{e \perp} / T_{e \|}$ at $\left.t=0\right)$ is plotted as a function of time for simulations with the parameters described in Sec. II with $T_{i} / T_{e \|}=5$ and $T_{e \perp} / T_{e \|}=1$ (solid line), $T_{e \perp} / T_{e \|}=1.5$ (dotted), and $T_{e \perp} / T_{e \|}=2$ (dashed). The results with NPIC are plotted in (a) and with CELESTE3D in (b). The temperatures are averaged over $x$ in the interval $-0.2 L \leqslant z \leqslant 0.2 L$ for NPIC and $-0.3 L \leqslant z \leqslant 0.3 L$ for CELESTE3D.

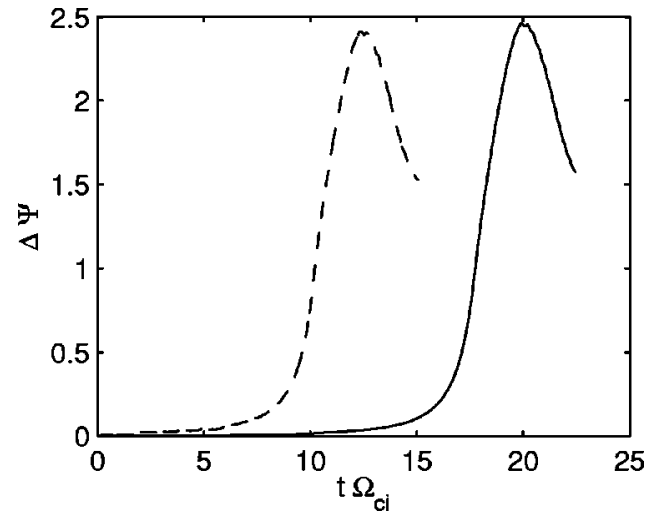

FIG. 13. The reconnected flux as a function of time is plotted for simulations in the $(x, z)$ plane with NPIC (solid) and CELESTE3D (dashed). The plasma parameters are as described in Sec. II, but with $\rho_{i} / L=3.656$.

whole domain. In Fig. 13, we show the evolution of the current sheet by plotting the reconnected flux $\Delta \Psi$ for the Harris sheet with half the current sheet thickness of the GEM challenge $\left(\rho_{i} / L=3.656\right)$ from the simulations. A domain size $L_{x} \times L_{z}=12.8 d_{i} \times 6.4 d_{i} \quad$ (corresponding to $L_{x} \times L_{z}=51.2 L$ $\times 25.6 L)$ is considered. Unlike the standard GEM challenge case without perturbation, reconnection involves the whole domain by $t \Omega_{c i} \approx 20$ for NPIC and by $t \Omega_{c i} \approx 13$ for CELESTE3D. It should be remarked that the fast reconnection phase is very similar in the two codes. The delayed start of the fast reconnection phase in NPIC is probably because more particles are used in NPIC than in CELESTE3D, which reduces the initial level of noise. ${ }^{23}$

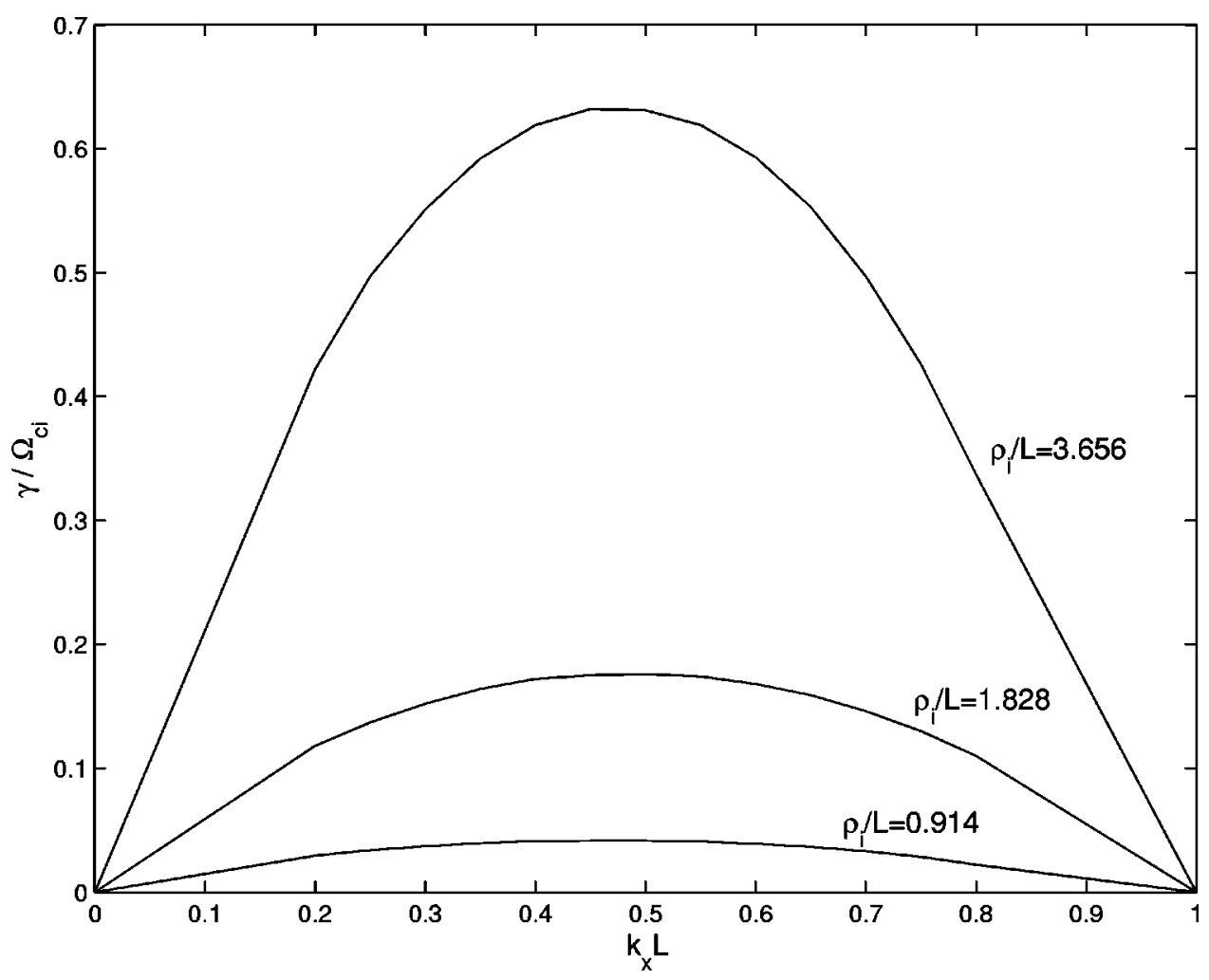

FIG. 12. The growth rate $\gamma / \Omega_{c i}$ of the tearing mode as a function of $k_{x} L$ is plotted for plasma parameters described in Sec. II and different values of $\rho_{i} / L$. 


\section{CONCLUSION AND DISCUSSION}

Reconnection onset is studied using results from two codes employing very different algorithms: NPIC is a massively parallel explicit code and CELESTE3D is an implicitmoment method PIC code. The results from NPIC and CELESTE3D complement and confirm each other. This degree of cross checking between codes, for which the GEM challenge is designed, is unusual. Because of their differences in resolution, the agreement between explicit and implicit results suggests that the important physical length scales involved in the onset of reconnection are comparable to or greater than the electron scales.

The simulations have pointed out the important role of the LHDI in reconnection onset. Our results confirm that the tearing instability saturates at a low level if no initial perturbation is added to the Harris equilibrium. Linear growth of the tearing instability is limited by increase in the parallel electron temperature, such that an anisotropy develops with $T_{e \perp} / T_{e \|}<1$ that strongly stabilizes the tearing mode. In three dimensions, the tearing instability does not saturate at small amplitudes. Because the mode spectrum reveals no significant oblique mode growth, the current sheet dynamics can be analyzed by studying the interrelationship between the current aligned instability that develops in the $(y, z)$ plane and the dynamics of the tearing modes in the $(x, z)$ plane. The analysis shows that the LHDI strongly modifies the linear and nonlinear evolution of the tearing instability. The LHDI causes a favorable electron temperature anisotropy, $T_{e \perp} / T_{e \|}>1$, and thins and peaks the current sheet. Both modifications are effective in enhancing the linear growth rate of the tearing instability. With a favorable anisotropy and a thin enough current sheet, the tearing instability grows large enough to decouple electrons and ions so that reconnection can encompass the whole domain.

In the current sheet considered there is no background plasma, the guide field is not present, and no normal component of the magnetic field is introduced. The influence of these on reconnection onset needs further, investigation.

Both laboratory and satellite observations ${ }^{32-34}$ have pointed out the presence of the LHDI near reconnection sites, but they have not identified a connection between this instability and reconnection through a contribution to anomalous resistivity.

Satellites have measured electron anisotropy during magnetic substorms. Shinohara et al..$^{34}$ observe electron anisotropy $\left(T_{e \perp} / T_{e \|}<1\right)$ at the substorm onset. A more comprehensive study of the electron anisotropy during reconnection has been performed by Birn et al. ${ }^{63}$ Through 1 year average data of satellite measurements, it is shown that an electron anistropy $\left(T_{e \perp} / T_{e \|}>1\right)$ precedes substorm onset. At onset, $T_{e \perp} / T_{e \|}<1$ is observed. After onset, $T_{e \perp} / T_{e \|}$ grows again. This behavior recalls closely what is observed in tearing simulations (see Fig. 11). Birn et al. ${ }^{63}$ also show that an ion temperature anisotropy is less relevant than an electron temperature anisotropy, and this also agrees with the simulations presented here.

It is not obvious how to verify current sheet thinning from observations. Our simulation starts with a plain Harris sheet from which the LHDI develops and thins the current sheet. In reality, the LHDI is always present in current sheets with an amplitude that corresponds to the saturation level for that particular current sheet. In particular, magnetic reconnection experiment results ${ }^{32,33}$ show a thinning of the current sheet, in conjunction with an enhancement of the LHDI amplitude. However, it is difficult to say if the enhancement of the LHDI is a cause or a consequence of the thinning process.

\section{ACKNOWLEDGMENTS}

The authors gratefully acknowledge useful discussions with J. Chen, B. Coppi, S. Hsu, J. Huba, H. Karimabadi, B. Rogers, and I. Shinohara.

This research is supported by the LDRD program at the Los Alamos National Laboratory, by the United States Department of Energy, under Contract No. W-7405-ENG-36 and by NASA, under the "Sun Earth Connection Theory Program" and the "Geospace Sciences Program."

${ }^{1}$ W. Gekelman, H. Pfister, Z. Lucky, J. Bamber, D. Leneman, and J. Maggs, Rev. Sci. Instrum. 62, 2875 (1991).

${ }^{2}$ M. Yamada, J. Geophys. Res. 104, 14529 (1999).

${ }^{3}$ J. Egedal, A. Fasoli, and J. Nazemi, Phys. Rev. Lett. 90, 135003 (2003).

${ }^{4}$ I. Furno, T. Intrator, E. Torbert et al., Rev. Sci. Instrum. 74, 2324 (2003).

${ }^{5}$ J. B. Taylor, Rev. Mod. Phys. 58, 741 (1986).

${ }^{6}$ M. Øieroset, T. D. Phan, M. Fujimoto, R. P. Lin, and R. P. Lepping, Nature (London) 412, 414 (2001).

${ }^{7}$ A. Nishida, Geomagnetic Diagnostics of the Magnetosphere (Springer, New York, 1978).

${ }^{8}$ E. R. Priest, Solar Magnetohydrodynamics (Reidel, Dordrecht, 1982).

${ }^{9}$ M. M. Romanova and R. V. E. Lovelace, Astron. Astrophys. 262, 26 (1992).

${ }^{10}$ E. G. Blackman, Astrophys. J. Lett. 456, LT87 (1996).

${ }^{11}$ H. Lesch and G. T. Birk, Astron. Astrophys. 324, 461 (1997).

${ }^{12}$ E. G. Harris, Nuovo Cimento 23, 115 (1962).

${ }^{13}$ J. Birn, J. F. Drake, M. A. Shay et al., J. Geophys. Res. 106, 3715 (2001).

${ }^{14}$ B. Coppi, G. Laval, and R. Pelat, Phys. Rev. Lett. 16, 1207 (1966).

${ }^{15}$ J. F. Drake and Y. C. Lee, Phys. Fluids 20, 1341 (1977).

${ }^{16}$ A. A. Galeev, in Magnetospheric Tail Dynamics, edited by A. Nishida (Reidel, London, 1982).

${ }^{17}$ D. Biskamp, R. Z. Sagdeev, and K. Shindler, Cosm. Electrodyn. 1, 297 (1970).

${ }^{18}$ B. U. O. Sonnerup, in Solar System Plasma Physics, edited by L. T. Lanzerotti, C. F. Kennel, and E. N. Parker (North-Holland, New York, 1979), Vol. III, p. 45.

${ }^{19}$ T. Terasawa, Geophys. Res. Lett. 10, 475 (1983).

${ }^{20}$ M. A. Shay and J. F. Drake, Geophys. Res. Lett. 25, 3759 (1998).

${ }^{21}$ M. A. Shay, J. F. Drake, B. N. Rogers, and R. E. Denton, J. Geophys. Res. 106, 3759 (2001).

${ }^{22}$ B. N. Rogers, R. E. Denton, and J. F. Drake, J. Geophys. Res. 108, 1111 (2003).

${ }^{23}$ P. Ricci, J. U. Brackbill, W. Daughton, and G. Lapenta, Phys. Plasmas 11, 4102 (2004).

${ }^{24}$ V. A. Sergeev, D. G. Mitchell, C. T. Russel, and D. J. Williams, J. Geophys. Res. 98, 17345 (1993).

${ }^{25}$ R. Pellat, F. Coroniti, and P. Pritchett, Geophys. Res. Lett. 18, 143 (1991).

${ }^{26}$ P. L. Pritchett, J. Geophys. Res. 99, 5935 (1994).

${ }^{27}$ K. B. Quest, H. Karimabadi, and M. Brittnacher, J. Geophys. Res. 101, 179 (1996)

${ }^{28}$ M. I. Sitnov, A. S. Sharma, P. N. Guzdar, and P. H. Yoon, J. Geophys. Res. 107, 1256 (2002).

${ }^{29}$ D. Biskamp, Magnetic Reconnection in Plasmas (Cambridge University Press, Cambridge, NY, 2000).

${ }^{30}$ M. Scholer, I. Sidorenko, C. H. Jaroschek, R. A. Treumann, and A. Zeiler, Phys. Plasmas 10, 3521 (2003).

${ }^{31}$ I. Shinohara and M. Fujimoto, Phys. Rev. Lett. (submitted).

${ }^{32}$ T. A. Carter, H. Ji, F. Trintchouk, M. Yamada, and R. M. Kulsrud, Phys. 
Rev. Lett. 88, 015001 (2002).

${ }^{33}$ T. A. Carter, M. Yamada, H. Ji, R. M. Kulsrud, and F. Trintchouk, Phys. Plasmas 9, 3272 (2002)

${ }^{34}$ I. Shinohara, T. Nagai, M. Fujimoto, T. Terasawa, T. Mukai, K. Tsuruda, and T. Yamamoto, J. Geophys. Res. 103, 20365, 1998.

${ }^{35}$ R. C. Davidson, N. T. Gladd, and C. Wu, Phys. Fluids 20, 301 (1977).

${ }^{36}$ J. U. Brackbill, D. W. Forslund, K. B. Quest, and D. Winske, Phys. Fluids 27, 2682 (1984).

${ }^{37}$ W. Daughton, Phys. Plasmas 10, 3103 (2003).

${ }^{38}$ G. R. Burkhart and J. Chen, Phys. Fluids B 1, 1578 (1989).

${ }^{39}$ R. Horiuchi and T. Sato, Phys. Plasmas 6, 4565 (1999).

${ }^{40}$ W. Daughton, Phys. Plasmas 9, 3668 (2002).

${ }^{41}$ G. Lapenta and J. U. Brackbill, Phys. Plasmas 9, 1544 (2002).

${ }^{42}$ G. Lapenta, J. U. Brackbill, and W. Daughton, Phys. Plasmas 10, 1577 (2003).

${ }^{43}$ G. Lapenta and J. U. Brackbill, Nonlinear Processes Geophys. 7, 151 (2000).

${ }^{44}$ B. Coppi, Astrophys. J. 273, L101 (1983).

${ }^{45}$ J. Chen and P. Palmadesso, Phys. Fluids 27, 1198 (1984).

${ }^{46}$ J. Chen and Y. C. Lee, Phys. Fluids 28, 2137 (1985).

${ }^{47}$ J. Chen and Y. C. Lee, Phys. Fluids 31, 2944 (1988).

${ }^{48}$ W. Daughton, G. Lapenta, and P. Ricci, Phys. Rev. Lett. (to be published).

${ }^{49}$ W. Daughton, Phys. Plasmas 6, 1329 (1999).
${ }^{50}$ R. Morse and C. Nielson, Phys. Fluids 14, 830 (1971).

${ }^{51}$ D. Forslund, Space Sci. Rev. 42, 3 (1985).

${ }^{52}$ J. U. Brackbill and D. W. Forslund, in Multiple Times Scales, edited by J. U. Brackbill and B. I. Cohen (Academic Press, Orlando, 1985), p. 271.

${ }^{53}$ H. X. Vu and J. U. Brackbill, Comput. Phys. Commun. 69, 253 (1992).

${ }^{54}$ P. Ricci, G. Lapenta, and J. U. Brackbill, J. Comput. Phys. 183, 117 (2002).

${ }^{55}$ P. Ricci, G. Lapenta, and J. U. Brackbill, Geophys. Res. Lett. 29, 2008 (2002).

${ }^{56}$ J. F. Drake and Y. C. Lee, Phys. Rev. Lett. 39453 (1977).

${ }^{57}$ M. M. Kuznetsova and L. M. Zelenyi, Plasma Phys. Controlled Fusion 32, 1183 (1990).

${ }^{58}$ H. Karimabadi, K. B. Quest, and W. Daughton, "Role of electron anisotropy in the onset of magnetic reconnection," Geophys. Res. Lett. (submitted).

${ }^{59}$ P. Ricci, G. Lapenta, and J. U. Brackbill, Geophys. Res. Lett. 31, L06801 (2004).

${ }^{60}$ D. V. Forslund and B. Coppi, Bull. Am. Phys. Soc. 13, 306 (1998).

${ }^{61}$ J. Ambrosiano, L. C. Lee, and Z. F. Fu, J. Geophys. Res. 91, 113 (1986).

${ }^{62}$ Y. Shi, L. C. Lee, and Z. F. Fu, J. Geophys. Res. 92, 12171 (1987).

${ }^{63}$ J. Birn, M. F. Thomsen, J. E. Borovsky, G. D. Reeves, D. J. McComas, R. D. Belian, and M. Hesse, J. Geophys. Res. 102, 2309 (1997). 